\title{
Bereavement care for family caregivers of neuro-oncology patients
}

\author{
Sue E. Morris ${ }^{1,2,3}$, Timothy S. Sannes ${ }^{1,2}$ \\ ${ }^{1}$ Department of Psychosocial Oncology and Palliative Care, Dana-Farber Cancer Institute, Boston, MA, USA; ${ }^{2}$ Department of Psychiatry, Brigham \\ and Women's Hospital, and Harvard Medical School, Boston, MA, USA; ${ }^{3}$ Department of Psychiatry, Boston Children's Hospital, Boston, MA, USA \\ Contributions: (I) Conception and design: All authors; (II) Administrative support: All authors; (III) Provision of study materials or patients: None; \\ (IV) Collection and assembly of data: None; (V) Data analysis and interpretation: None; (VI) Manuscript writing: All authors; (VII) Final approval of \\ manuscript: All authors. \\ Correspondence to: Sue E. Morris, PsyD. Department of Psychosocial Oncology and Palliative Care, Dana-Farber Cancer Institute, 450 Brookline \\ Avenue, JF8, Boston, MA 02215, USA. Email: sue_morris@dfci.harvard.edu.
}

\begin{abstract}
Bereavement care is best conceptualized as a preventive model of care. It is an integral component of palliative care where support for the family begins at the time of diagnosis and continues beyond the death of the patient. Even though grief is a normal response to loss, the death of a loved one is believed to be the most powerful stressor in everyday life with the potential to cause great distress in all those closely associated with the deceased. In neuro-oncology, where patients often face limited prognoses, knowledge about approaches to bereavement support is particularly important. Despite this, research into the experience of bereaved caregivers is limited. As such, an opportunity exists to identify ways to help family caregivers not only cope with the death of their loved one but also to help families prepare for the death ahead of time. In this article, we offer guidelines about how best to support family caregivers before and after the death of the patient, drawing on the palliative care and bereavement literature.
\end{abstract}

Keywords: Bereavement; grief; palliative care; caregivers; neuro-oncology

Submitted Mar 19, 2020. Accepted for publication May 26, 2020.

doi: 10.21037/apm-20-708

View this article at: http://dx.doi.org/10.21037/apm-20-708

\section{Introduction}

Bereavement is a complex, multidimensional process that involves the physical, psychological, sociological and cultural domains of the human experience (1). The death of a loved one has long been recognized as the most powerful stressor in everyday life (2), which has important implications for how health professionals care for bereaved individuals. Even though grief is considered to be a normal and expected response to loss, bereaved individuals are at heightened risk of serious physical and mental health problems, including cardiovascular disease, substance use, depression and suicide (3). Coping following a significant loss is influenced by many factors, including the individual's personality, the relationship with the deceased, the circumstances surrounding the death and the social and cultural context in which bereavement is experienced $(3,4)$. While the majority of bereaved individuals adjust to the death of a loved one without requiring professional intervention, it is estimated that between 10-20\% experience prolonged grief or other psychiatric disorders (5-8).

Bereavement care is best conceptualized as a preventive model of care (9). It is considered a central component of high-quality end-of-life care by the hospice and palliative care movement (10-13) where support of the family begins at the time of diagnosis and extends beyond the death of the patient. Even though the development of bereavement services has lagged behind that of other elements of palliative care, the importance of providing specialized bereavement services to bereaved families is endorsed by 
the two national palliative care organizations in the USA. The National Hospice and Palliative care Organization (12) and the National Consensus project for Quality Palliative Care (13) offer clinical practice guidelines, including the assessment of bereavement risk and the provision of bereavement support. Research shows that hospice and palliative care services are associated with improved family reported quality of end-of-life care $(14,15)$, improved family satisfaction $(16,17)$, and better bereavement outcomes for family members (18-21).

In neuro-oncology, there is very limited research investigating the bereavement needs of family caregivers following the death of a patient (22). Given the high rate of patient deaths and the high level of caregiver burden throughout the illness, integrating interdisciplinary palliative care early in the disease trajectory is essential to support both patients and their caregivers during treatment and also to improve bereavement outcomes. In this article, we examine the literature addressing the bereavement experiences of caregivers of neuro-oncology patients and offer guidelines about bereavement support drawing from both the palliative care and bereavement literature.

\section{The experience of caregivers of neuro-oncology patients across the disease trajectory}

Primary brain and central nervous system (CNS) cancers affect both children and adults and represent a substantial source of morbidity and mortality worldwide (23). While brain tumors are rare compared to other malignancies, in many incidences they result in severe physical and cognitive disability and have higher mortality rates overall (24). Patients with high-grade primary malignant glioma, for example, face a terminal prognosis early in their illness and must negotiate profound and often devastating physical, cognitive and behavioral changes. These changes not only include a decline in functioning, but can also include substantial changes in patients' personalities and behaviors. The high mortality and dramatic changes in patients can understandably place significant burden on family caregivers.

Prior to a patient's death, family caregivers typically experience high levels of stress associated with the uncertainty of their loved one's prognosis, protracted treatment, high caregiving demands, as well as the neurocognitive deficits and personality changes commonly arising from the tumor and its treatment (25). Existential distress is considered to be an important component of burden among family caregivers (26). According to Applebaum et al. (26) who conducted a systematic review of 35 studies examining existential distress in caregivers of patients with brain tumors, themes of identity; isolation; responsibility and guilt; death anxiety; deriving meaning and personal growth; and spirituality and religion were identified. Across this review, studies consistently pointed to distress emerging early in the disease course and remaining unmet by healthcare providers. Also, in relation to prognostic awareness, while patients are often unaware of their life expectancy (27), caregivers can find themselves in the middle of knowing the importance of this information and wanting to protect their loved one from this oftendevastating information (28).

The literature also identifies several risk factors that have been linked to increased distress in family caregivers. These risk factors include younger age, greater economic burden, unmet needs, and lower levels of social support, spirituality and caregiver mastery (29). Schubart et al. (30), for example, in a study of 25 family caregivers of adult patients with brain tumors found that the caregivers provided extensive uncompensated care, completing tasks that not only were physically and emotionally demanding but required them to solve problems and make decisions as care needs changed, for which they felt untrained and unprepared. They concluded that because caregiver information needs are often emergent and not always known at the time of a clinic visit, physicians need to improve the delivery of information while also providing psychosocial support (30).

Taken together, the often-unwavering caregiving burden and distress that many family caregivers experience during the course of the patient's illness and treatment, have the potential to greatly impact their subsequent grief and bereavement following the patient's death.

\section{The nature of grief}

Following a significant death, grief can best be described as the intense emotional and physical reaction that an individual experiences, where attachment is considered key (31). Typically, grief follows a wave-like pattern and is characterized by deep sadness and yearning or pining to be with the deceased again (32,33). Even though grief is a normal reaction to a significant loss, for many it remains an intensely painful period where adjustments can take months or even years (3).

From a psychological perspective, grief involves adapting to change brought about by loss $(33,34)$. As a consequence 
of the death, a bereaved individual not only loses the person but also the many other things that person represented, ranging from practical roles to their hopes and dreams for the future. In serious illness, many bereaved individuals also speak of losing their job as a "caregiver", which is relevant in neuro-oncology where the caregiving burden is high and time away from the patient is limited. Part of bereavement support is to help the bereaved individual regain a sense of control in their life and restore balance.

An individual's worldview and expectations also play an important role in their bereavement experience (4,34). Most people expect that children will outlive their parents and that adults will live long and healthy lives. When someone dies prematurely or unexpectedly even within the context of a cancer diagnosis, many basic assumptions about the world are challenged, often resulting in a discrepancy between what was expected to happen and what actually happened. The greater the discrepancy, the more challenging it can be for the bereaved individual to adjust to the death of a loved one. This is one reason why the death of a child is considered to be the most difficult loss a person can bear because it challenges our beliefs about the way we think life and death "should" be (34). Western society also maintains a view that the death of a loved one is something bereaved individuals should "get over" quickly. Such beliefs reflect the "fix-it" mentality of our society where the bereaved are expected to return to normal in the same way they would recover from a common cold. This view of grief is inaccurate as it implies that there is a linear relationship between time and progress. Rather, for any bereaved person, grief tends to follow a wave-like pattern punctuated by periods of intensified emotion, often for years to come, where the strength and frequency of the waves gradually lessens over time, as the loss becomes integrated into their lives.

The dual process model of coping with bereavement (35) conceptualizes normal grief as an oscillation between confronting the loss (loss orientation) and compartmentalizing it so that the bereaved individual can deal with changes in their life as a result of the death (restoration orientation). Stroebe and Schut (35) view the central mechanism as the confrontation-avoidance mechanism in which a balance between the two poles needs to be achieved after a major loss. Yearning for the deceased or replaying the patient's final days are examples of loss orientation stressors and mastering new skills or contemplating one's changed identity are examples of restoration stressors. For many caregivers, especially spouses and parents who have provided the majority of care to their partner or child, building a new life can be particularly challenging given that their social networks have often shrunk as the demands of caregiving increased. Bereavement support following the death of a patient from a serious illness, therefore, needs to specifically target helping the individual gradually re-engage in social activities, while also dealing with any painful memories of what they witnessed as their loved one's health deteriorated.

\section{Bereavement research}

Recent research has focused on the diagnosis, treatment and prevention of prolonged grief disorder (PGD) and other psychiatric disorders following the death of a loved one (6,36-38). PGD and persistent complex bereavement disorder are terms currently used in the literature to describe the debilitating set of symptoms reported by those with a problematic adjustment to a loss. PGD has been included in ICD-11 as a disorder specifically associated with stress (39) and persistent complex bereavement disorder is a category of further study in DSM-5 (32). Prolonged grief affects $2-3 \%$ of the general population and approximately $10 \%$ of bereaved individuals (7), with a prevalence of $10-20 \%$ after the death of a romantic partner (36-38,40,41). For bereaved parents the rates are even higher $(42,43)$. The primary symptoms in these diagnostic categories are a state of intense yearning for the deceased and an inability to accept the reality of the loss $(32,36,39)$. Feelings of guilt are also common where the focus is usually associated with regrets related to the deceased or aspects of their care (37).

In a bereavement survey conducted at our cancer center (44), bereaved family members were asked to describe the ways in which the clinical team was helpful in dealing with their loss. The findings indicated that bereaved family members most often reported compassionate care by the team, which incorporated communication that conveyed empathy, caring and concern for both the patient and caregiver. Bereavement outreach from the providers, including the offering of condolences, was also found to be helpful. When asked what they wished the clinical team could have done prior to their loved one's death that would have helped them in dealing with their loss, the most common themes were provide more accurate information about prognosis, the end-of-life period and the dying process, earlier hospice referral and more caregiver support.

A number of risk factors for worse bereavement outcomes have been identified in the literature [e.g., 
Table 1 Risks factors for worse bereavement outcomes

Lower levels of social support

History of psychiatric disorders, such as depression and substance use

Concurrent stressors, including greater economic burden

Younger age

Previous losses

Unexpected diagnosis

High initial distress

Death of a child

Sudden death

Hospital-based death

$(3,21,42,45)]$, and are presented in Table 1 . Identifying those individuals at risk of a difficult bereavement is an essential task of the psychosocial or palliative care clinician even though the lack of a systematic process to identify those individuals can be challenging (46). Screening family caregivers throughout the course of the patient's illness and implementing psychological support prior to the death of the patient is believed to mitigate complicated or prolonged grief reactions (13).

\section{Bereavement research specific to neuro- oncology}

Bereavement research within neuro-oncology is limited and supportive interventions for bereaved caregivers are lacking (47), despite brain cancer being identified as one of the top three diseases for which caregivers receive bereavement counseling once enrolled in hospice (20). A recent systematic review of bereaved caregivers of patients with high-grade glioma (HGG) produced only four qualitative articles published from 2004 to 2015 that met their eligibility criteria, which included caregivers of patients diagnosed aged 18 and over and addressed their post bereavement experience (22). Piil et al. (22) found that bereaved family caregivers expressed profound grief and had unmet needs and questions related to the disease and their caregiving role. Further, they experienced depressive symptoms and fatigue that impacted their work and social life. The authors concluded that research is needed to identify how bereaved caregivers of people with HGG prefer to be supported.
An earlier study conducted by Schubart et al. (30), which included a subset of six bereaved family caregivers of patients with brain tumors, found that all bereaved caregivers regretted not being better prepared to manage symptoms during the end-of-life period and not knowing what to expect. They also regretted not accepting hospice care sooner. Similarly, more recently, Piil and Jarden (47) conducted an exploratory qualitative study with eight bereaved caregivers of patients with HGG. They identified several themes of caregiving, including that late-stage caregiving is comprehensive and taxing, that caregivers experienced both feelings of grief after the patient died and a sense of relief, and that caregivers felt that they would have benefited from audio recordings of important information discussed during consultations. After the patient's death, the caregivers suggested that a bereavement consultation with the professionals they knew would help them clarify questions about the patient's end-of-life care and facilitate reflections about their caregiving role.

\section{Palliative care model}

Bereavement is an important component of palliative care where the care of the patient and family throughout the course of the patient's illness directly impacts the bereavement experience of the family. Figure 1 outlines the "Palliative Care Model", where disease directed therapy and interdisciplinary palliative care are provided concurrently. Central is the goal of addressing the patient and the family's physical, psychological, social, and spiritual needs combined with treatments targeting the underlying disease process. As a terminal disease progresses, care that is focused on comfort, pain management, and decreasing other burdensome symptoms plays a larger and larger role. Throughout the patient's care, hope plays an important role, where what is hoped for transitions as the hope for cure, life extension or a miracle moves toward hope for comfort and meaning at the end of life.

Neuro-oncology patients, and thereby their family members, may have less access to specialized palliative care compared to other types of cancer (49). As a result, many neuro-oncologists often serve in the role of supporting patient and families' goals of care, leading to what has been termed a neuropalliative approach (50). This approach, while also extending to neurodegenerative diseases and neurologic diseases with slower rates of progression compared to many brain cancers, honors front-line clinicians' need to incorporate advanced care planning, 


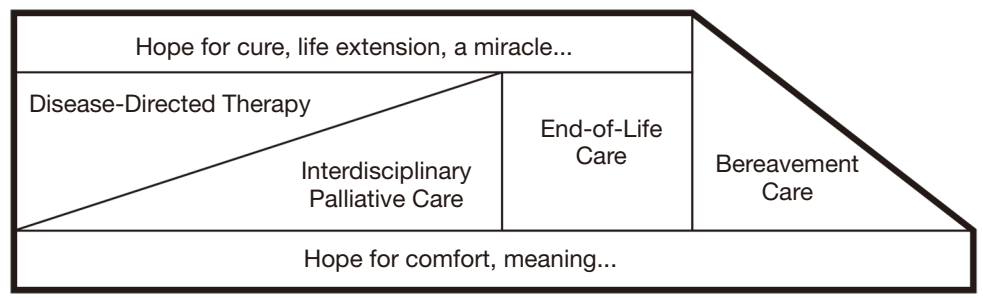

Figure 1 Palliative care model. Adapted with permission from Liben, Papadatou \& Wolfe (48).

discussion about prognosis and supporting family caregivers throughout the trajectory of illness, including bereavement. Indeed, qualitative work has echoed the importance of early support for caregivers (51), and some clinics have prioritized this caregiver support as a standard of practice offering individualized assessments and tailored care plans (52). While coordination of caregiver support to this level has inherent challenges, caregivers identify this as central to their well-being. Opportunities for support, according to Collins et al. (53), involves navigation between providers, the provision of individualized information and proactive emotional support at different stages, and early integration of palliative care services to facilitate support for caregivers. Building upon this, the same group proposed a preliminary framework of supportive and palliative care interventions for HGG to guide care for patients and their families at different transition points within the illness trajectory (54). They outline several principles that underpin the framework, including: (I) ensure that care and services are aligned with patient/family caregiver needs according to the transition point in the illness, (II) care should be proactive, and (III) care should include routine bereavement support. For each transition point in care from the time of the initial diagnosis to after the patient's death, they identify needs and gaps and describe in detail the corresponding service components.

Specific to bereavement, several studies have demonstrated the positive impact of palliative care and the enrolment in hospice on bereavement outcomes. For example, Bradley et al. (18) examined depression in bereaved caregivers and found that caregivers of patients who were enrolled in hospice for three or fewer days were significantly more likely to experience major depressive disorder 6 to 8 months after the death of their loved one than those caregivers whose loved ones had longer hospice enrollments. Similarly, Wright et al. (21) found that the family caregivers of cancer patients who died in the hospital were significantly more likely to suffer from
PGD than those caregivers of patients who died at home with hospice. More recently, Higgins et al. (55) found that caregivers' assessment of quality of end-of-life care, and in turn bereavement, was related to the use of hospice and the place of death, which they argue are potentially modifiable factors.

\section{Caring for the bereaved family caregivers}

The literature describing the experience of family caregivers of neuro-oncology patients provides important insight into avenues for increased support of family caregivers during the treatment phase that in turn, can potentially lead to better bereavement outcomes. We recommend that clinicians and teams play an active role in assessing the family caregivers before and after the death of the patient. The following guidelines listed in Table 2 are recommended, adapting them as necessary to the cultural context of the family.

\section{Psychological strategies for helping the bereaved}

Bereavement care comes in many forms, including individual counseling, peer group support and structured group programs facilitated by psychosocial clinicians. A range of organizations offer bereavement support, such as hospices, nonprofit community organizations, and faith-based groups, in addition to informal care offered by individual providers, such as primary care physicians. As a result, the types of interventions and support that bereaved caregivers receive vary greatly according to the setting in which the patient received care at the end of life and the provider's discipline, experience and orientation (9). Bereavement care is one of the core services provided by hospice under the federal Conditions of Participation from the Centers for Medicare and Medicaid Services (CMS). Under these CMS regulations, hospices provide 
Table 2 Considerations for bereavement support before and after a patient's death

Before death

Acknowledge and respond to emotion that caregivers typically experience immediately following a diagnosis

Provide written information about the tumor at different time points

Assign a care coordinator who can check in regularly, screen for distress and answer immediate questions or concerns of patients/family caregivers

Clinicians should adopt a proactive stance rather than a reactive stance to issues

Provide caregivers with accurate information about the disease trajectory and what to expect, which is especially relevant at different time points in the illness trajectory, including at the time of diagnosis, following a recurrence and during the end-of-life period, including the dying process

Encourage family caregivers to pay attention to their own physical and mental health needs given how stressful caregiving can be, including seeing their doctor or a therapist

Create individual opportunities for family caregivers to meet with psychosocial clinicians to facilitate conversations about unspoken fears as well as concerns about preparing for the death of their loved one

Offer caregiver support groups that create a safe place for caregivers to share their stories and seek guidance

For caregivers with risk factors for difficult bereavement outcomes, make a referral to a community based mental health clinician for additional support prior to the patient's death

Involve palliative care early in the disease trajectory as a part of usual care, as recommended by the ASCO clinical practice guidelines

Make an early referral to hospice to maximize support for both patient and family

Check in with caregivers after the patient has been discharged to hospice to lessen feelings of abandonment

After death

Offer condolences to family caregivers as a routine part of care, sending a sympathy card or note of condolence

Make a bereavement phone call to check in with the family, to say 'goodbye' or make recommendations for support as needed

Offer opportunities for family caregivers to return to the hospital at a later date to meet with the team to have questions answered

Provide annual neuro-oncology memorial services for staff and families to come together to remember patients who have died in the previous year

As part of bereavement follow-up, determine scheduled outreach for ongoing assessment (e.g., at 6 and 12 months post-death), screening for bereavement risk where possible

Offer bereavement support groups specific to neuro-oncology and the type of relationship loss, e.g., spouse or parent group, to enhance a sense of connection and lessen feelings of isolation

Provide referrals to community-based bereavement services for individual and group support as appropriate, including referrals for children and teenagers to counselors and organizations specializing in child bereavement

support to family members for 13 months following the death of a loved one (12). Hospices also generally provide bereavement support to the community even if the person who died wasn't enrolled in hospice.

Irrespective of whether the bereaved caregivers engage in hospice bereavement programs, in the initial months of their bereavement, it is not uncommon for family caregivers to reach out to the team or institution where their loved one received care for support. Sometimes, a family member might want to meet with the team to seek answers to lingering questions or they might want to visit to express their appreciation and say "goodbye". This is often the case in neuro-oncology where the relationship with the caregiver and team can be particularly close. Providing opportunities for these types of meetings can be extremely beneficial to their bereavement, especially as bereaved caregivers often replay over and over the events of their loved one's last weeks and second guess their actions. Being able to address their questions or concerns and acknowledge the extent of their caregiving role can provide much needed reassurance 
and validation.

Research findings about the effectiveness of psychological interventions for normal and complicated grief are mixed, where findings vary according to the study design and the type of interventions targeted. Effective treatments for complicated grief based on cognitive-behavioral models show promise $(37,38)$, including Internet-based therapistassisted interventions to prevent PGD (56) and manualized interventions targeting family dysfunction (57).

When working with the bereaved either individually or in a group setting, the overall aims of bereavement support are: (I) to help the bereaved adapt to life without the deceased, and (II) to help them maintain a connection with their loved one that is now based on memory and legacy (34). Ideally, psychosocial clinicians should meet with bereaved family members individually prior to joining a group to assess their functioning and readiness for a group and to gain an understanding of the circumstances surrounding the death.

Routine issues to be addressed include:

(I) The bereaved's story, paying attention to the burden of caregiving and what they have witnessed;

(II) What they have lost with the death of their loved one;

(III) Their social, cultural and religious/spiritual backgrounds;

(IV) Their social support network;

(V) Past psychiatric history, including depression and substance use;

(VI) Suicidal ideation;

(VII) Concurrent stressors, e.g., financial difficulties;

(VIII) Prior losses and coping skills;

(IX) Unresolved or unfinished business with the deceased;

(X) Their expectations and goals for seeking support.

Adopting an education, guidance and support model of bereavement care (9), strategies to help the bereaved based on self-help principles and cognitive behavior therapy $(33,34,58)$ can be grouped into seven categories as shown in Table 3. When parents of young children die, memory and legacy activities are especially important. Children also benefit from attending community-based organizations that specialize in child bereavement, including bereavement camps, where they can meet other children who have experienced similar losses.

\section{Barriers to integrating bereavement services}

There are several barriers that impede bereaved caregivers accessing bereavement support following the death of a loved one. These include lack of comprehensive hospitalwide bereavement programs, difficulty identifying the bereaved, late hospice referrals and limited access or utilization of services. Few hospitals, cancer centers, and nursing homes offer bereavement services for the families of their patients and if even if they do, identifying the bereaved can be difficult $(9,59)$. As a result, the bereaved typically have to seek out support on their own, which can be challenging for many who are often overwhelmed by their grief, reluctant to do so (60), unsure about where to go (61), or whether the services will help (11).

A particular challenge for bereavement care following the death of a patient from a neuro-oncology disease is the timing of a hospice referral. In our experience, if a patient is referred late to hospice and the length of stay is short, caregivers are less willing to participate in hospice bereavement programs because they do not feel a strong connection with the hospice providers. As we have previously highlighted, hospice enrollment and preventing a patient from dying in the hospital are related to better bereavement outcomes. However, in many cases caregivers prefer to return to where their loved one was treated, which is not always practical or possible, especially if bereavement programs are not offered or they live some distance away. Given that bereaved individuals diagnosed with PGD have been found to underutilize mental health services, it is strongly recommended that caregivers be connected with services while the patient is still alive (60).

\section{Recommendations for future research}

Research with bereaved caregivers is an evolving field, with substantial gaps remaining in neuro-oncology. As noted above, continuing to target barriers to care for the bereaved is an important area of future research. Interventions, while promising, will continue to struggle with retention if such barriers are not addressed. Innovative approaches that engage caregivers throughout the disease trajectory and into their bereavement using technology show promise. Examples include online communities, in which caregivers can share their perspectives (62), in addition to online bereavement support groups and internet-based therapist-assisted interventions to 
Table 3 Psychological strategies to help the bereaved based on self-help and cognitive behavior therapy principles

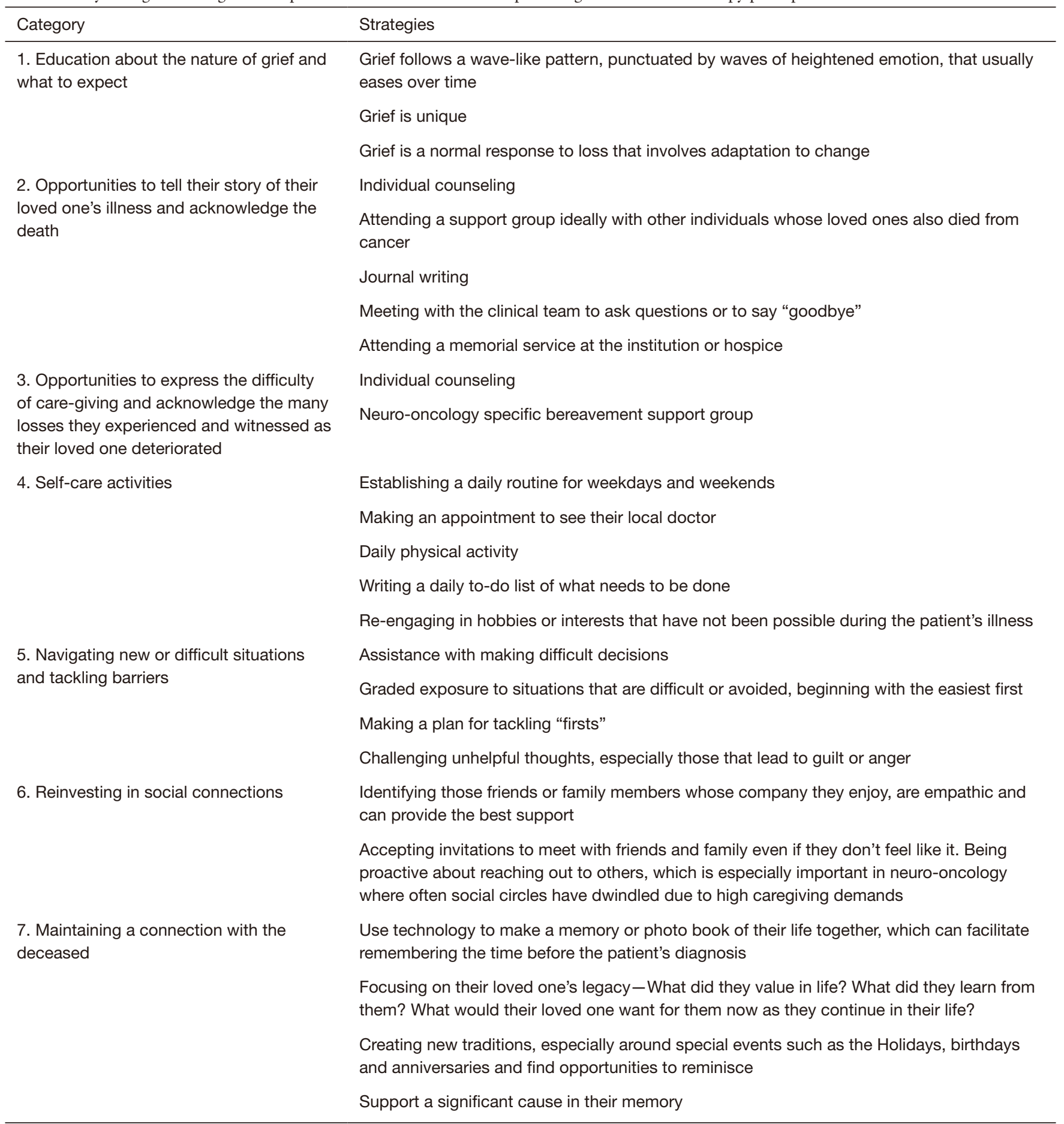

prevent PGD (56). Not only are online interventions and other telehealth technologies cost effective and scalable but they are also attractive because they can eliminate barriers to seeking care, such as distance, time constraints and impaired mobility.

\section{Conclusions}

In neuro-oncology, family caregivers typically cope with high levels of stress that are associated with uncertainty and changing caregiver demands, in addition to witnessing 
over time, neurocognitive deficits and personality changes in their loved ones. Together, these stressors have the potential to greatly impact their bereavement experience. Given that bereavement care is best considered a preventive model of care, a strong argument needs to be made to integrate interdisciplinary palliative care at the time of a neuro-oncology diagnosis to support both the patient and their family throughout treatment and beyond, consistent with recent expert consensus practice guidelines about oncology care (63). Increased attention needs to be paid to developing programs that tailor support for caregivers during the patient's treatment, including the provision of accurate information about their loved one's prognosis and psychosocial support from the outset. Early hospice referrals and the development of hospital bereavement programs are critical as research has repeatedly demonstrated benefit to bereaved family caregivers from these programs as they potentially reduce barriers to accessing bereavement care, while creating opportunities to connect with other bereaved caregivers, following the death of the patient.

\section{Acknowledgments}

We would like to thank the many bereaved family members who have shared their stories with us over the years.

Funding: None.

\section{Footnote}

Provenance and Peer Review: This article was commissioned by the Guest Editors (Jerome Graber, Hany Soliman) for the series "Palliative Care in Neuro-Oncology" published in Annals of Palliative Medicine. The article has undergone external peer review.

Conflicts of Interest: Both authors have completed the ICMJE uniform disclosure form (available at http://dx.doi. org/10.21037/apm-20-708). The series "Palliative Care in Neuro-Oncology" was commissioned by the editorial office without any funding or sponsorship. SEM reports to receive royalties from her self-help book, Overcoming grief: a selfhelp guide using cognitive behavioural techniques. The other author has no conflicts of interest to declare.

Ethical Statement: The authors are accountable for all aspects of the work in ensuring that questions related to the accuracy or integrity of any part of the work are appropriately investigated and resolved.
Open Access Statement: This is an Open Access article distributed in accordance with the Creative Commons Attribution-NonCommercial-NoDerivs 4.0 International License (CC BY-NC-ND 4.0), which permits the noncommercial replication and distribution of the article with the strict proviso that no changes or edits are made and the original work is properly cited (including links to both the formal publication through the relevant DOI and the license). See: https://creativecommons.org/licenses/by-nc-nd/4.0/.

\section{References}

1. Sanders CM. Risk factors in bereavement outcome. In: Stroebe MS, Stroebe W, Hansson RO. editors. Handbook of Bereavement: Theory, Research, and intervention. Cambridge, Cambridge University Press, 1999:255-67.

2. Holmes TH, Rahe RH. The social readjustment rating scale. J Psychosom Res 1967;11:213-8.

3. Stroebe M, Schut H, Stroebe W. Health outcomes of bereavement. Lancet 2007;370:1960-73.

4. Malkinson R. Cognitive grief therapy: constructing a rational meaning to life following loss. New York: Norton, 2007.

5. Aoun SM, Breen LJ, Howting DA, et al. Who needs bereavement support? A population based survey of bereavement risk and support need. PLoS One 2015;10:e0121101.

6. Lobb EA, Kristjanson LJ, Aoun SM, et al. Predictors of complicated grief: A systematic review of empirical studies. Death Stud 2010;34:673-98.

7. Lundorff M, Holmgren H, Zachariae R, et al. Prevalence of prolonged grief disorder in adult bereavement: A systematic review and meta-analysis. J Affect Disord 2017;212:138-49.

8. Prigerson HG. Complicated grief: when the path of adjustment leads to a dead-end. Bereave Care 2004;23:38-40.

9. Morris SE, Block SD. Adding value to palliative care services: The development of an institutional bereavement program. J Palliat Med 2015;18:915-22.

10. Agnew A, Manktelow R, Taylor BJ, et al. Bereavement needs assessment in specialist palliative care: A review of the literature. Palliat Med 2010;24:46-59.

11. Cherlin EJ, Barry CL, Prigerson HG, et al. Bereavement services for family caregivers: How often used, why, and why not. J Palliat Med 2007;10:148-58.

12. National Hospice and Palliative Care Organization. Guidelines for bereavement care in hospice. Alexandria: NHPCO, 2018.

13. National Consensus Project for Quality Palliative Care. 
Clinical practice guidelines for quality palliative care. 4th Edition, Richmond, National Coalition for Hospice and Palliative Care, 2018. Available online: https://www. nationalcoalitionhpc.org/ncp

14. Wachterman MW, Pilver C, Smith D, et al. Quality of end-of-life care provided to patients with different serious illnesses. JAMA Intern Med 2016;176:1095-102.

15. Wright AA, Keating NL, Ayanian JZ, et al. Family perspectives on aggressive cancer care near the end of life. JAMA 2016;315:284-92.

16. Gelfman LP, Meier DE, Morrison RS. Does palliative care improve quality? A survey of bereaved family members. J Pain Symptom Manage 2008;36:22-8.

17. Roza KA, Lee EJ, Meier DE, et al. A survey of bereaved family members to assess quality of care on a palliative care unit. J Palliat Med 2015;18:358-65.

18. Bradley EH, Prigerson H, Carlson MDA, et al. Depression among surviving caregivers: Does length of hospice enrollment matter? Am J Psychiatry 2004;161:2257-62.

19. Christakis NA, Iwashyna TJ. The health impact of health care on families: A matched cohort study of hospice use by decedents and morality outcomes in surviving, widowed spouses. Soc Sci Med 2003;57:465-75.

20. Palmer WW, Yuen FK. The impact of hospice patient disease type and length of stay on caregiver utilization of grief counseling: A 10-year retrospective study. Am J Hosp Palliat Care 2017;34:880-6.

21. Wright AA, Keating NL, Balboni TA, et al. Place of death: correlations with quality of life of patients with cancer and predictors of bereaved caregivers' mental health. J Clin Oncol 2010;28:4457-64.

22. Piil K, Nordentoft S, Larsen A, et al. Bereaved caregivers of patients with high-grade glioma: A systematic review. BMJ Support Palliat Care 2019;9:26-33.

23. GBD 2016 Brain and Other CNS Cancer Collaborators. Global, regional, and national burden of brain and other CNS cancer, 1990-2016: A systematic analysis for the Global Burden of Disease Study 2016. Lancet Neurol 2019;18:376-93.

24. Rees JH. Diagnosis and treatment in neuro-oncology: an oncological perspective. Br J Radiol 2011;84:S82-9.

25. Ownsworth T, Goadby E, Chambers SK. Support after brain tumor means different things: family caregivers' experiences of support and relationship changes. Front Oncol 2015;5:33.

26. Applebaum AJ, Kryza-Lacombe M, Buthorn J, et al. Existential distress among caregivers of patients with brain tumors: a review of the literature. Neurooncol Pract
2016;3:232-44.

27. Diamond EL, Prigerson HG, Correa DC, et al. Prognostic awareness, prognostic communication, and cognitive function in patients with malignant glioma. Neuro Oncol 2017;19:1532-41.

28. Applebaum AJ, Buda K, Kryza-Lacombe M, et al. Prognostic awareness and communication preferences among caregivers of patients with malignant glioma. Psychooncology 2018;27:817-23.

29. Sherwood PR, Cwiklik M, Donovan HS. Neuro-oncology family caregiving: review and directions for future research. CNS Oncol 2016;5:41-8.

30. Schubart JR, Kinzie MB, Farace E. Caring for the brain tumor patient: family caregiver burden and unmet needs. Neuro Oncol 2008;10:61-72.

31. Bowlby J. Attachment and loss. New York: Basic Books, 1980.

32. American Psychiatric Association. Diagnostic and statistical manual of mental disorders. 5 th edition. Arlington: American Psychiatric Publishing, 2013.

33. Morris SE. Overcoming grief: a self-help guide using cognitive behavioural techniques. London: Robinson, 2018.

34. Morris SE. Block SD. Grief and bereavement. In: Grassi L, Riba M. editors. Clinical Psycho-oncology: An international perspective. Oxford: Wiley-Blackwell, 2012:271-80.

35. Stroebe M, Schut H. The dual process model of coping with bereavement: Rationale and description. Death Stud 1999;23:197-224.

36. Prigerson HG, Horowitz MJ, Jacobs SC, et al. Prolonged grief disorder: Psychometric validation of criteria proposed for DSM-V and ICD-11. PLoS Med 2009;6:e1000121. Erratum in: PLoS Med 2013;10. doi:10.1371/annotation/ a1d91e0d-981f-4674-926c-0fbd2463b5ea.

37. Shear MK. Complicated grief. N Engl J Med 2015;372:153-60.

38. Shear MK, Reynolds CF 3rd, Simon NM, et al. Optimizing Treatment of Complicated Grief: A Randomized Clinical Trial. JAMA Psychiatry 2016;73:685-94.

39. ICD-11. International Classification of Diseases for Mortality and Morbidity Statistics. Eleventh Revision, World Health Organization. Available online: http://CD.who.int

40. He L, Tang S, Yu W, et al. The prevalence, comorbidity and risks of prolonged grief disorder among bereaved Chinese adults. Psychiatry Res 2014;219:347-52.

41. Kersting A, Brahler E, Glaesmer H, et al. Prevalence of complicated grief in a representative population-based sample. J Affect Disord 2011;131:339-43.

42. Morris S, Fletcher K, Goldstein R. The Grief of Parents 
After the Death of a Young Child. J Clin Psychol Med Settings 2019;26:321-38.

43. Goldstein RD, Lederman RI, Lichtenthal WG, et al. The grief of mothers after the sudden unexpected death of their infants. Pediatrics 2018;141:e20173651.

44. Morris SE, Nayak MM, Block SD. Insights from Bereaved Family Members About End-of-Life Care and Bereavement. J Palliat Med 2020. [Epub ahead of print]. doi:10.1089/jpm.2019.0467.

45. Mash HB, Fullerton CS, Shear MK, et al. Complicated grief and depression in young adults: personality and relationship quality. J Nerv Ment Dis 2014;202:539-43.

46. Morris SE, Anderson CM, Tarquini SJ, et al. A standardized approach to bereavement risk-screening: a quality improvement project. J Psychosoc Oncol 2020;38:406-17.

47. Piil K, Jarden M. Bereaved caregivers to patients with high-grade glioma: A qualitative explorative study. J Neurosci Nurs 2018;50:94-9.

48. Liben S, Papadatou D, Wolfe J. Paediatric palliative care: challenges and emerging ideas. Lancet 2008;371:852-64.

49. Walbert $T$. Integration of palliative care into the neurooncology practice: patterns in the United States. Neurooncol Pract 2014;1:3-7.

50. Creutzfeldt CJ, Kluger BM, Holloway RG. Neuropalliative Care: A Guide to Improving the Lives of Patients and Families Affected by Neurologic Disease. 1st edition. Springer, 2019.

51. Cavers D, Hacking B, Erridge SE, et al. Social, psychological and existential well-being in patients with glioma and their caregivers: a qualitative study. CMAJ 2012;184:E373-82.

52. Page MS, Chang SM. Creating a caregiver program in neuro-oncology. Neurooncol Pract 2017;4:116-22.

53. Collins A, Lethborg C, Brand C, et al. The challenges and suffering of caring for people with primary malignant glioma: qualitative perspectives on improving current

Cite this article as: Morris SE, Sannes TS. Bereavement care for family caregivers of neuro-oncology patients. Ann Palliat Med 2021;10(1):953-963. doi: 10.21037/apm-20-708 supportive and palliative care practices. BMJ Support Palliat Care 2014;4:68-76.

54. Philip J, Collins A, Brand C, et al. A proposed framework of supportive and palliative care for peope with high-grade glioma. Neuro Oncol 2018;20:391-9.

55. Higgins PC, Garrido MM, Prigerson HG. Factors predicting bereaved caregiver perception of quality of care in the final week of life: Implications for health care providers. J Palliat Med 2015;18:849-57.

56. Litz BT, Schorr Y, Delaney E, et al. A randomized controlled trial of an internet-based therapist-assisted indicated preventive intervention for prolonged grief disorder. Behav Res Ther 2014;61:23-34.

57. Kissane DW, Zaider TI, Li Y, et al. Randomized controlled trial of family therapy in advanced cancer continued into bereavement. J Clin Oncol 2016;34:1921-7.

58. Kavanagh DJ. Towards a cognitive-behavioural intervention for adult grief reactions. Br J Psychiatry 1990;157:373-83.

59. Kusano AS, Kenworthy-Heinige T, Thomas CR. Survey of bereavement practices of cancer care and palliative care physicians in the Pacific Northwest United States. J Oncol Pract 2012;8:275-81.

60. Lichtenthal WG, Nilsson M, Kissane DW, et al. Underutilization of mental health services among bereaved caregivers with prolonged grief disorder. Psychiatr Serv 2011;62:1225-9.

61. Lichtenthal WG, Corner GW, Sweeney CR, et al. Mental health services for parents who lost a child to cancer: If we build them, will they come? J Clin Oncol 2015;33:2246-53.

62. Lucas MR. A private caregiver listServ: Maximum benefit for minimum cost. J Psychosoc Oncol 2011;29:168-74.

63. Ferrell BR, Temel JS, Temin S, et al. Integration of palliative care into standard oncology care: American Society of Clinical Oncology clinical practice guideline update. J Clin Oncol 2017;35:96-112. 